\title{
Periodontitis And Cerebrovascular Disease
}

\author{
Prejna Nair ${ }^{1}$, Dr.Radhika Arjunkumar ${ }^{2}$ \\ (Intern, Saveetha Dental College, Saveetha university, Chennai) \\ (Senior Lecturer,Department of Periodontics,Saveetha Dental College,Saveetha University,Chennai)
}

\begin{abstract}
Systemic health is often associated with the condition of the oral cavity because many systemic diseases manifest in the mouth. Effects of systemic disorders on periodontal diseases are well established. Periodontal disease affects many systemic diseases like cardiovascular diseases, diabetes, pre-term low birth weight babies, preclampsia, respiratory infections, cancer, rheumatoid artritis, gastrointestinal disease , osteoporosis, renal disease, prostatitis, alzheimer's disease and cerebrovascular disease. With an increasing body of epidemiological and ecperimental work, specific risk factors and risk indicators for periodontis such as tobacco smoking,demographic factors, and socio-econonomic status have been identified and acknowledged.Any changes to the systemic health because of any of these factors can be seen as a change in periodontal health. So, any changes in the systemic health can definetely affect the periodontal health and vice verca.Periodontitis patients are susceptible to cerebrovascular diseases and many other diseases. This review article mainly highlights the connection between periodontitis and cerebrovascular disease.
\end{abstract}

Keywords: Cerebrovascular disease,Focal infection,Periodontal pockets, Periodontitis, Systemic disorders

\section{Introduction}

Periodontitis is a disease of inflammation and infection of the periodontal ligaments and alveolar bone that supports the tooth structure. Periodontitis results from complex interplay between chronic bacterial infections and the inflammatory host response leading to irreversible destruction of tooth structure causing loss of the tooth. [2]This disease harbors large numbers of anaerobic gram negative pathogens, including Porphyromonas Gingivalis and Aggregatibacter Actinomycetemcomitans.Periodontal disease can effect a person's susceptibilty for systemic diseases in many ways,shared risk factors like smoking,stress,aging,race or ethnicity and gender. The dynamics of periodontium are a product of its circulation, hormonal changes and mechanism of immune responses.[3]

Cerebrovascular disease is a disease of blood vessels that supplies the brain. Cerebrovascular disease can mainly be divided into embolism and aneurysum. Most important risk factors that can cause cerebrovascular diseases are smoking, demographic factors,socio-economic status. Causes of cerebrovascular disease can be periodontitis, [2]obesity, diabetes, hypertension and etc.

\section{PERIODONTIUM}

Periodontium is a reservoir of cytokines like tumor necrosis factor-a,interlukin-1B, and gamma interferon. So, a spillover of these mediators can enter the circulation and induce systemic diseases,such as cerebrovascular disease. [3]Periodontitis happens when there is inflammation and infection of the periodontal ligaments and alveolar bone that supports the tooth structure., harmful oral bacteria freely enters the blood stream and forms plaque and fatty deposits in blood vessels and travels throughout the organs of the body causing pathological manifestation. [4]

\section{PERIODONTITIS AND CEREBROVASCULAR DISEASE}

Bacteria that initiates periodontal disease are called periopathogens.most common bacteria that is seen in periodontitis are p.gingivalis and a.actinomycetemcomitans. Highly vascularized and ulcerarated tissues of the oral cavity are highly susceptible to invasion of periopathogens which in order will enter the blood stream and causes a condition called bacteremia. Bacteria can induce thrombus formation by platelet agregation degrading collagen.Bacteria can enter the blood stream and organs through a few pathways and cause focal infection.[3]

\section{PATHWAYS CAUSING FOCAL INFECTION}

\section{A)Metastatic infection from oral cavity due to bacteremia}

Often,bacteremias are short lived and transient, highest intensity would be the first 30 mins after the trigger episode.they are dealt swiftly by body defenses.This may lead to spreading of the organism to different target organs like kidney, liver and brain causing subclinical, acute and chronic infections which can cause many critical conditions. 
B)Metastatic injury caused by oral microbial toxins

The pathogenecity of a bacteria depends upon the structural component of the cells for example, capsules,fimbria,lipopolysaccharides or active secretion of the substances, where the microorganism damage the host tissues or protects the bacteria against host defenses. These bacterial toxins are carried by the vascular or lymphatic system thereby causing cytotoxic effects at tissue sites causing a large number of pathological manifestation.

C)Metastatic inflammation due to immunological injury caused by microorganisms in the oral cavity

The microorganisms enters the blood instantly during bacteremia and react with the antibodies and form complexes to cause many acute and chronic inflammatory reactions where they are deposited.

\section{PERIODONTAL POCKETS}

Periodontal pockets are pathologically deepened gingival sulcus.Bacteria from the periodontal pockets can enter the bloodstream during activities like mastication and brushing teeth.Infections and low grade inflammatory processes influence the development of artherosclerosis and ischaemic lesions. [5] Dental sites with deep pockets harbor a large number of bacteria with a positive correlation between pocket probing depth and bacterial levels during ischemic stroke in the present study. A greater incidence of ischemic stroke was previously observed in young patients with periodontal disease, particularly in those with decreased numbers of remaining teeth.[6]

\section{HEMORRHAGIC CEREBROVASCULAR EVENT}

Another mechanism for atherogenesis is the induction of immunological processes leading to increased levels of C-reactive proteins(CRP) and increases in CRP even within the range of normal values is considered a reliable predictor of cardiovascular disease. In present studies, 13 out of 20 patients develop ischaemic stroke linked to periodontitis developing a hemorrhagic cerebrovascular event.[3]
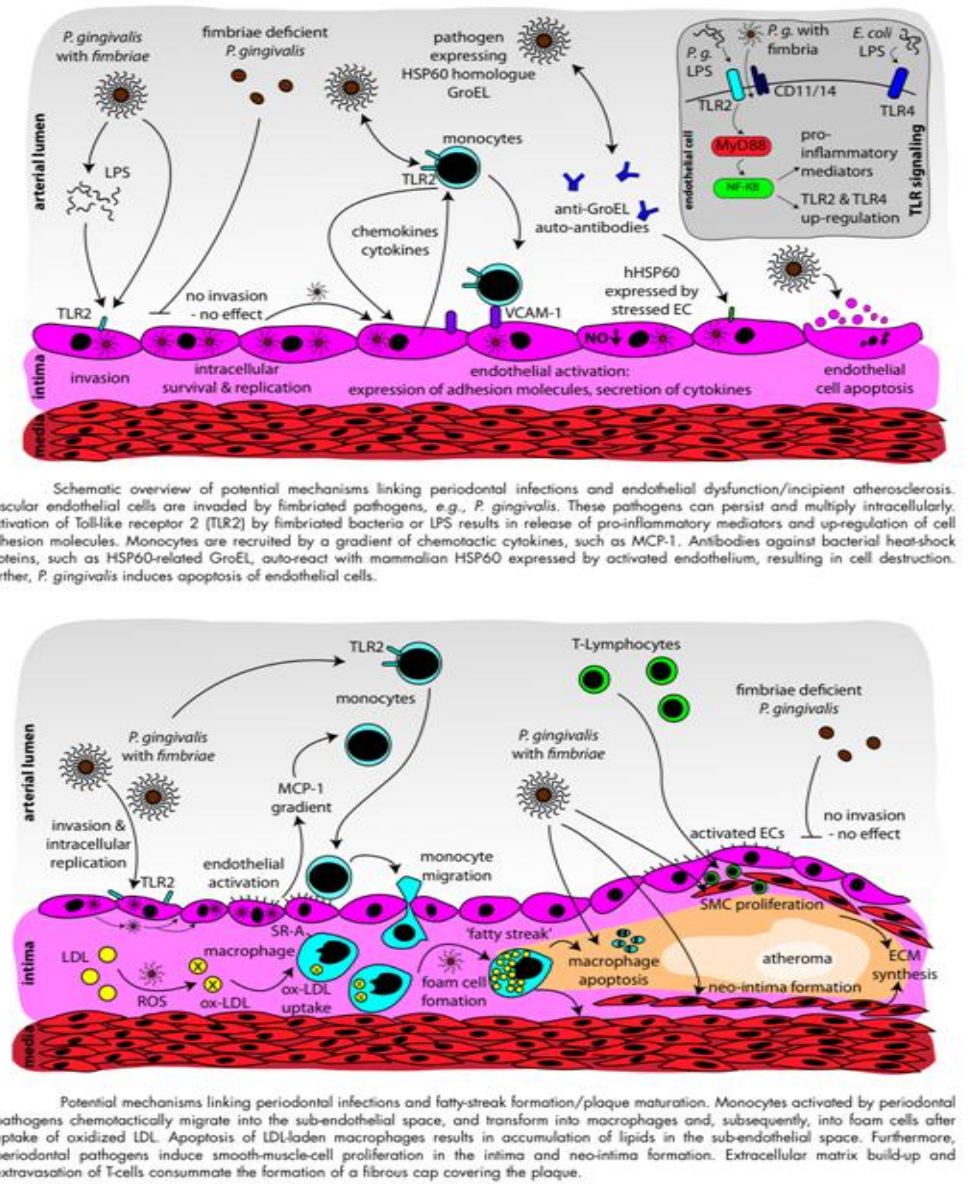


\section{Conclusion}

Periodontal disease is a paradigm of chronic infection in dental pathology which can lead to many systemic manifestation,likecerebrovascular disease. Poor oral hygiene, many other shared factors, like smoking, diabetes, genetic factors combined, can lead to systemic disorders. Oral cavity is one system which is interconnected with the whole body. A healthy oral cavity prevents from numerous systemic condition.

\section{References:}

[1] P. Lopez- Jornet,J.D Berna-Mestre,J.D Berna-Serna,F.Camacho-Alonso,S.Fernandez-Millan and M. Reus-Pintado Measurement of Artherosclerosis Markers in Patients with Periodontitis:a case control study: J Periodontal 2012;83:690-698

[2] Snophia Suresh,Satya Narayana,P.Jayakumar,Uma Sudhakar and V.Pramod Evaluation of anti-inflammatory effect of statins in chronic periodontitis : Indian journal of pharmocology.2013jul-aug;45(4):391-394.

[3] Minkle Gulati,Vishal Anand,Nikil Jain, Bhargavi Anand, Rohit Bahuguna, Vivek Govila and Pavitra Rastogi Essentials of Periodontal Medicine in Preventive Medicine: Int J Prev Med.2013 September;4(9):988-994

[4] Ghizoni J.S, Luis antonio de Assis Taveira,Gustavo Pompermaier Garlet,Marcos Flavio Ghizoni,Jefferson Ricardo Pereira,Thiago Jose Dionisio,Daniel Thomas Brozoski,Carlos Ferreira Santos, Adriana Campos Passanezi Sant'Ana Increased Levels of Porphyromonas Gingivalis are Associated With Ischemic and Hemorrhagic Cerebrovascular Disease in Humans:an in vivo study : J.Appl.Oral Sci. Vol.20 no.1 Bauru Jan./Feb.2012

[5] M.Kebschull, R.T. Demmer, and P.N Papapanou "Gum Bug,leave my heart alone!"-Epidermologic and Mechanistic Evidence Linking Periodontal Infections and Artherosclerosis J.Dent Res89(9):879-902,2010

[6] Armin J.Grau,Heiko Becher,Christoph M.Ziegler,Christoph Lichy, Florian Buggle,Claudia Kaiser,Rainer Lutz,Stefan Bultman, Micheal Preusch and Christof E.Dorfer Periodontal Disease as a Risk Factor For Ischemic Stroke :Journal of the american Heart Association STROKE.2004;35:496-501; originally published online January 5, 2004

[7] Dnisia Haba,Silvia Teslaru,Didona Ungureana,Diana Hodorog,C.Alecu,Anagabriela,Bengchiac,L.Zetu,Codrina Ancuta,E.Ancuta,A.Nemtoi,Cristina Evaluation of serum and gingival crevicular fluid C-reactive protein and IL-6 in patients with periodontitis and transient ischemic attacks : Romanian Journal of morphology \& embryology 2011,52(4);1243-1247

[8] Jorge H Ramirez, Roger M Arce, Adolfo Contreras Periodontal Treatment Effects on Endothelial Function and Cardiovascular Disease Biomarkers in Subject With Chronic Periodontitis: Protocol For a Randomized Clinical Trial Ramirez et al.Trials 2011,12:46 